\title{
Conveniently Accessible Polymer Nanoparticles of Adjustable Polarity
}

\author{
Brigitte Korthals, Maria Carmen Morant-Miñana, Christiane Hohberger, and Stefan Mecking* \\ Chair of Chemical Materials Science, Department of Chemistry, University of Konstanz, \\ 78464 Konstanz, Germany
}

\begin{abstract}
The postpolymerization modification by hydroformylation of 1,2 polybutadiene nanoparticles provides access to colloidally stable aqueous dispersions of $<20 \mathrm{~nm}$ particles. Their polarity can be adjusted via the degree of conversion of double bonds over a broad range. Fluorescence studies illustrate the polarity of the environment experienced by pyrene as a probe molecule.
\end{abstract}

\section{Introduction}

Polymer nanoparticles are of broad academic and industrial interest. Examples of specific topics of interest are the introduction and transport of guest molecules such as poorly soluble drugs, carriers in aqueous multiphase catalysis, or homogeneous incorporation of functional molecules into solid materials. ${ }^{1}$ In many cases, nanoscale entities of $<30 \mathrm{~nm}$ size are desirable. Also, the presence of the particles as aqueous dispersions is required.

Both dendrimers and polymer aggregates have been studied as hosts for small molecules due to their ability to form amphiphilic structures with an apolar interior and a polar periphery. ${ }^{1 \mathrm{a}} 1 \mathrm{~d}, 1 \mathrm{~g}$ The multistep synthesis for preparing dendrimers, as well as the strong dependence of size and structure of polymer aggregates on external conditions like concentration or temperature, can be a limitation. An alternative are polymer nanoparticles prepared by heterophase polymerization. ${ }^{2}$ Free-radical microemulsion polymerization affords particles as small as $510 \mathrm{~nm}$, albeit it must be noted that for a given monomer amenable to classical free radical emulsion polymerization this procedure can not necessarily be adopted to microemulsion conditions straightforwardly. ${ }^{3}$ Catalytic polymerizations are largely complementary to free-radical techniques in terms of the polymers accessible, and enable

*To whom correspondence should be addressed. Fax: +497531 885152 Tel: + 49753188 5151. E mail: stefan.mecking@uni konstanz.de.

(1) (a) Caruso, F. Colloids and Colloid Assemblies; Wiley-VCH: Weinheim, 2004 (b) Haag, R. Angew. Chem. 2004, 116, 280 284. Haag, R. Angew. Chem., Int. Ed. 2004 43, 278 282. (c) Bosman, A. W ; Janssen, H. M.; Meijer, E. W. Chem. Rev. 1999, 99 , 1665 1688. (d) Grayson, S. M.; Frechet, J. M. J. Chem. Rev. 2001, 101, 38193868. (e) Cölfen, H. Macromol. Rapid Commun. 2001, 22, 219 252. (f) Allen, T. M.; Cullis, P. R. Science 2004, 303, 1818 1822. (g) Broz, P.; Driamov, S.; Ziegler, J.; Ben-Haim, N.; Marsch, S.; Meier, W.; Hunziker, P. Nano Lett. 2006, 6, 2349 2353. (h) Kunna, K.; Müller, C.; Loos, J.; Vogt, D. Angew. Chem. 2006, 118, 7447 7450. Kunna, K.; Müller, C.; Loos, J.; Vogt, D. Angew. Chem., Int. Ed. 2006, 45, 72897292.

(2) (a) Distler, D Wässrige Polymerdispersionen; VCH: Weinheim, 1999. (b) Lovell, P. A.; El-Aasser, M. S. Emulsion Polymerization and Emulsion Polymers; Wiley: Chichester, U.K., 1997. (c) Urban, D.; Takamura, K. Polymer Dispersions and their Industrial Applications. Wiley-VCH: Weinheim, 2002. (d) van Herk, A. M. Chemistry and Technology of Emulsion Polymerisation; Blackwell Publishing: Oxford, 2005. (e) Charleux, B.; Ganachaud, F. Polymerization in Aqueous Dispersed Media. In Macromolecular Engineering; Matyjaszewski, K., Gnanou, Y., Leibler, L., Eds.; Wiley-VCH: Weinheim, 2007; Vol. 1, pp 605-642.

(3) (a) Kuo, P. L.; Turro, N. J.; Tseng, C. M.; El-Aasser, M. S.; Vanderhoff, J. W. Macromolecules 1987, 20, 1216 1221. (b) Feng, L.; Ng, K. Y. S. Macromolecules 1990, 23, 1048 1053. (c) Ferrick, M. R.; Murtagh, J.; Thomas, J. K. Macromolecules 1989, 22, 1515 1517. (d) Perez-Luna, V.H.; Puig, J. E.; Castano, V. M.; Rodriguez, B. E.; Murthy, A. K.; Kaler, E. W. Langmuir 1990, 6, 1040 1044. (e) Antonietti, M.; Basten, R.; Lohmann, S. Macromol. Chem. Phys. 1995, 196, 441 466. (f) Pavel, F. M. J. Dispersion Sci. Technol. 2004, 25, 1. (g) Capek, I.; Juranicová, V.; Barton, J.; Asua, J. M.; Ito, K. Polym. Int. 1997, 43, 1 7. (h) Loh, S.-E.; Gan, L.-M.; Chew, C.-H.; Ng, S.-C. J. Macromol. Sci., Pure Appl. Chem. 1995, 32, 16811697. polymer microstructure control. ${ }^{4}$ Very small polymer particles are accessible with water-soluble catalyst precursors, or by polymerization in microemulsion. ${ }^{5,6}$ Thus, aqueous dispersions of 1,2-polybutadiene nanoparticles of $15 \mathrm{~nm}$ average size are accessible. We now report on a straightforward route to colloidally stable very small polymer nanoparticles of broadly variable polarity, by postpolymerization modification ${ }^{7}$ of these particles, for which the vinyl groups of 1,2-polybutadiene are well suited.

\section{Experimental Section}

General Methods and Materials. Dihydrogen (99.999 vol. \%) and a 1:1 mixture of $\mathrm{CO}\left(99.997\right.$ vol. \%) and $\mathrm{H}_{2}(99.999$ vol. \%) were purchased from Air liquide. Pyrene (99\%, sublimed; Aldrich) and triphenylphosphine (Fluka) were used as received. Toluene was deoxygenated by destillation under argon. Millipore grade water was used for fluorescence measurements.

Differential scanning microscopy (DSC) was carried out on a Netzsch F1 instrument at a heating/cooling rate of $10 \mathrm{~K}$ min ${ }^{1}$ on approximately $5 \mathrm{mg}$ of polymer $\left(T_{\mathrm{m}}\right.$ given are from the second heating cycles). Molecular weights were determined with gel per meation chromatography (GPC) in $1,2,4$ trichlorobenzene at $160^{\circ} \mathrm{C}$ on a Polymer Laboratories 220 instrument equipped with Mixed Bed PL columns. Data reported refers to universal calibration.

Transmission electron microscopy (TEM) was carried out on a Zeiss Libra 120 instrument operated at $120 \mathrm{kV}$ acceleration voltage. Polymer dispersions of ca. $0.02 \mathrm{wt}$. \% concentration were applied to a carbon coated grid and dried.

For scanning force microscopy (AFM) freshly cleaved mica was used without further treatment, alternatively glass substrates previously cleaned with a 7:3 mixture of 96 wt. $\% \mathrm{H}_{2} \mathrm{SO}_{4}$ and 30 wt. $\% \mathrm{H}_{2} \mathrm{O}_{2}$ were employed. A drop of dispersion was placed on the resting substrate, which was then accelerated at a rate of $300 \mathrm{rpm} \mathrm{s}{ }^{1}$ to a final speed of $2000 \mathrm{rpm}$. The height of the particles was determined with a JPK NanoWizard atomic force microscope in the intermittent contact mode using a Silicon tip

(4) (a) Mecking, S. Colloid Polym. Sci. 2007, 285, 605 619. (b) Claverie, J. P.; Soula, R. Prog. Polym. Sci. 2003, 28, 619.

(5) (a) Kolb, L.; Monteil, V.; Thomann, R.; Mecking, S. Angew. Chem. 2005, 117, 433-436. (b) Kolb, L.; Monteil, V.; Thomann, R.; Mecking, S. Angew. Chem., Int. Ed. 2005, 44, 429 432. (c) Gottker-Schnetmann, I.; Korthals, B.; Mecking, S. J. Am. Chem. Soc. 2006, 128, 77087709 .

(6) Monteil, V.; Wehrmann, P.; Mecking, S. J. Am. Chem. Soc. 2005, 127, 1456814569 .

(7) (a) Bastero, A.; Mecking, S. Macromolecules 2005, 38, 220 222. (b) Leube, H. F.; Schmidt-Thümmes, J.; Zeller, E.; Maas, H. (BASF), WO 99/28357, 1999. [Chem. Abstr. 1999, 131, 19491f] (c) Zeller, E.; Leube, H. F.; Schlarb, B.; Kneuper, H.-J.; Roeper, M. (BASF), EP 0810236, 1997 [Chem. Abstr. 1998, 128, 48657h] (d) Chemtob, A.; Heroguez, V.; Gnanou, Y. Macromol. Rapid Commun. 2005, 26, 17111715 
with a force constant of $40 \mathrm{~N} \mathrm{~m}{ }^{1}$ and resonant frequency of about $300 \mathrm{kHz}$. Height, amplitude and phase images were recorded simultaneously.

Dynamic light scattering (DLS) was performed on a Malvern NanoZS ZEN 3600 particle sizer ( $173^{\circ}$ backscattering) on diluted dispersions. The autocorrelation function was analyzed using the Malvern dispersion technology software 5.1 algorithm to obtain volume weighted particle size distributions.

Infrared spectra of the isolated polymer were recorded on a Perkin Elmer Spectrum 100 instrument with an attenuated total reflection (ATR) sampling accessory. For a calibration curve, dispersions of 1,2 polybutadiene and of completely converted, hydroformylated polymer were mixed in several weight ratios. The IR spectra were recorded directly on the dispersions to hinder separation of the two different particle types; water was accounted for by a background correction. The peaks at 905 and $1720 \mathrm{~cm}{ }^{1}$ were integrated and the ratio of the areas were plotted against the weight ratio.

Hydroformylation Procedure. Polybutadiene dispersions were prepared according to [6]. Hydroformylations were carried out in a mechanically stirred $285 \mathrm{~mL}$ pressure reactor equipped with a heating/cooling block controlled by a thermocouple dip ping into the reaction mixture. In a typical hydroformylation, $10 \mathrm{~g}$ of latex (6 wt. \% polymer, $9.6 \mathrm{wt}$ \% sodium dodecyl sulfate (SDS)) was diluted with $20 \mathrm{~g}$ of water and degassed by carefully applying vacuum and flushing with argon. $5.2 \mathrm{mg}(0.020 \mathrm{mmol})$ of $\left[\mathrm{Rh}(\mathrm{CO})_{2}(\mathrm{acac})\right]^{8}$ and $21 \mathrm{mg}(0.080 \mathrm{mmol})$ of triphenylphosphine were dissolved in $1 \mathrm{~mL}$ of toluene under argon and added to the latex. The mixture was transferred to the pressure reactor under an argon atmosphere, pressurized with $1: 1 \mathrm{CO} / \mathrm{H}_{2}$, and heated under stirring to the respective temperature. After $20 \mathrm{~h}$ the reaction was stopped by cooling and releasing the pressure.

The latices were dialyzed for 5 days against neat water (Spectra/ Por Dialysis Membrane with a molecular weight cutoff of 6 8,000), reconcentrated, filtered and 2,6 di tert butyl 4 methyl phenol (BHT) was added as a radical inhibitor. The long term colloidal stability of the hydroformylated dispersions is lower in comparison to the polybutadiene dispersion, which possibly is due to a lower compatibility of the apolar hydrocarbon chain of the SDS with the polymer. For analyses of the bulk polymer, an aliquot was precipitated with excess methanol, filtered, washed with water and methanol and dried in vacuo at $50{ }^{\circ} \mathrm{C}$.

Fluorescence Studies. For fluorescence measurements $5.5 \mu \mathrm{L}$ of a toluene stock solution of pyrene $\left(480 \mathrm{mg} \mathrm{L}^{1}\right.$ ) were added to $3 \mathrm{~mL}$ of the respective latex. After evaporation of the toluene and dilution of the samples $(0.7 \mathrm{~mL}$ with $2.3 \mathrm{~mL}$ of water $)$ fluorescence emission spectra were recorded with a Perkin Elmer LS 50 fluorimeter (bandwidth: $2.5 \mathrm{~nm}$, scanning speed $100 \mathrm{~nm}$ min ${ }^{1}$ ) at room temperature in cuvettes with a $1 \mathrm{~cm}$ path length. The samples were excited at $333 \mathrm{~nm}$. As expected, excimer emission was not observed due to the low concentration of pyrene employed for the measurement $\left[1.30^{5} \times 10^{8} \mathrm{~mol} \mathrm{~L}{ }^{1}\right]$. Very dilute polymer dispersions were employed to avoid saturation of the emission spectra, scattering by the polymer particles, as well as destabilization of the nanoparticles. The water soluble quencher diethylaminoethanol (DAE) optionally added does not affect the colloidal stability of the nanoparticles. Even high DAE concen tration lead to no observable change in DLS traces. DAE is an effective quencher for pyrene in water, indeed no significant fluorescence signal is observed in aqueous pyrene solution when $\mathrm{DAE}$ is present in the concentration used for the determination of the $\mathrm{I}_{3} / \mathrm{I}_{1}$ ratios.

\section{Results and Discussion}

Hydroformylation allows for the introduction of polar carbonyl moieties, employing simple reagents $\left(\mathrm{H}_{2}, \mathrm{CO}\right)$ an unreacted excess of which can also be easily removed. Hydroformylations of

(8) Shapley, J. R. Inorg. Synth. 2004, 34, 127.
Scheme 1. Hydroformylation of 1,2 Polybutadiene Dispersions
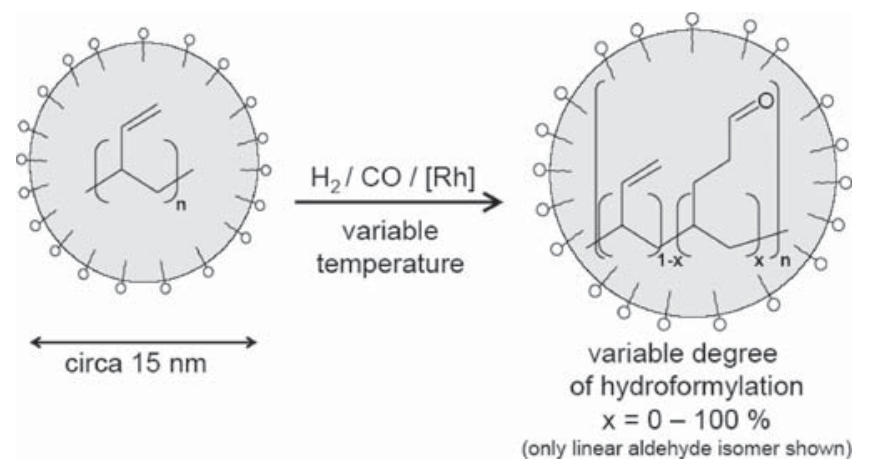

unsaturated polymers in organic solution have been well studied, albeit full conversion of the double bonds were difficult to achieve, likely due to gel formation by aldol condensation especially at higher conversions. 9,10

Colloidally stable aqueous dispersions of $15 \mathrm{~nm}$ nanoparticles of semicrystalline, syndiotactic polybutadiene (97\% 1,2 and 3\% 1,4-cis as determined by IR spectroscopy; ${ }^{11} M_{\mathrm{n}} 3.4 \times 10^{4} \mathrm{~g} \mathrm{~mol}{ }^{1}$; $\left.M_{\mathrm{w}} / M_{\mathrm{n}} 2.1\right)$ were prepared by catalytic microemulsion polymerization of butadiene. For hydroformylation in the nanoparticles, to a 1,2-polybutadiene dispersion of typically $2 \mathrm{wt}$. $\%$ polymer solids content was added $\left[\mathrm{Rh}(\mathrm{CO})_{2}(\mathrm{acac})\right] / \mathrm{PPh}_{3}(\mathrm{P}: \mathrm{Rh} 4: 1$ molar ratio), and the dispersion was exposed to $\mathrm{H}_{2} / \mathrm{CO}$ (1:1) pressure (Scheme 1, Table 1).

Hydroformylation is evidenced by the observation of the $v_{\mathrm{CO}}$ band at $1720 \mathrm{~cm}^{1}$ in IR spectra of the isolated polymers (Figure 1). The reduction of the intensity of the band at 905 $\mathrm{cm}^{1}$ (deformation characteristic of the vinyl group) provides an estimate of the degree of conversion of the double bonds. Partial hydroformylation occurred even at mild conditions of only $40{ }^{\circ} \mathrm{C}$ and 20 bar (entry 1). Temperature proved to be a convenient parameter to control the degree of hydroformylation. Varying the reaction temperature in the range from 40 to $60{ }^{\circ} \mathrm{C}$ afforded partially functionalized polymer particles with ca. 10 to $80 \%$ degree of functionalization (entries 2 to 4). At elevated temperature and pressure $\left(80{ }^{\circ} \mathrm{C}\right.$ and 60 bar) essentially complete conversion of the double bonds occurred.

Also under these harsher reaction conditions, colloidal stability was not affected and the particles retained their identity, that is no agglomeration occurred, as evidenced by dynamic light scattering (DLS; cf. Table 1 and Supporting Information for details). ${ }^{12}$ In order to estimate the significance of hydrogenation as a side reaction, 1,2-polybutadiene dispersions were exposed to hydrogen pressure only under conditions otherwise identical to the aforementioned hydroformylation studies. At $40{ }^{\circ} \mathrm{C}$ and 20 bar $\mathrm{H}_{2}$ the degree of hydrogenation was below the detection limit, that is, less than $3 \%$, as determined by ${ }^{1} \mathrm{H}$ NMR. At $80{ }^{\circ} \mathrm{C}$ and 60 bar of $\mathrm{H}_{2}$ (that is twice the $\mathrm{p}\left(\mathrm{H}_{2}\right)$ vs the maximum $\mathrm{H}_{2}$ partial pressure in the hydroformylation studies) after $20 \mathrm{~h}$ reaction time a degree of hydrogenation of ca. $50 \%$ was observed. This illustrates that

(9) McGrath, M. P.; Sall, E. D.; Tremont, S. J. Chem. Rev. 1995, 95, 381398. (10) (a) Sanui, K.; MacKnight, W. J.; Lenz, R. W. Macromolecules 1974, 7, 952 954. (b) Azuma, C.; Mitsuboshi, T.; Sanui, K.; Ogata, N. J. Polym. Sci.: Polym. Chem. Ed. 1980, 18, 781 797. (c) Mills, P. L.; Tremont, S. J.; Remsen, E. E. Ind. Eng. Chem. Res. 1990, 29, 1443 1454. (d) Tremont, S. J.; Remsen, E. E.; Mills, P. L. Macromolecules 1990, 23, 19841993.

(11) Morero, D.; Santambrogio, A.; Porri, L.; Ciampelli, F. Chim. Ind. 1959, 41, 758762 .

(12) In the case of the fully modified polyaldehyde particles a $\mathrm{P} / \mathrm{Rh}$ molar ratio of $8: 1$ was required to hinder partial agglomeration to $40 \mathrm{~nm}$ particles. A possible explanation is that under these conditions hydroformylation is slower, and crosslinking occurs sufficiently fast to hinder aggregation of the initially soft particles. 
Table 1. Synthesis Parameters ${ }^{a}$ and Properties of Nanoparticle Dispersions

\begin{tabular}{|c|c|c|c|c|c|c|}
\hline entry & $\begin{array}{c}\text { temperature of } \\
\text { hydroformylation }\left({ }^{\circ} \mathrm{C}\right)\end{array}$ & $\begin{array}{c}\text { pressure of } \\
\text { hydroformylation (bar) }\end{array}$ & $\begin{array}{c}\text { degree of } \\
\text { conversion }^{b}(\%)\end{array}$ & $\operatorname{size}^{c}(\mathrm{~nm})$ & $\begin{array}{l}\text { surface tension }^{d} \\
\qquad(\mathrm{mN} / \mathrm{m})\end{array}$ & $\begin{array}{c}T_{\mathrm{m}} \text { of bulk }{ }^{e} \\
\left({ }^{\circ} \mathrm{C}\right)\end{array}$ \\
\hline 1 & n.a. & n.a. & 0 & 13 & 60 & 154 \\
\hline 2 & 40 & 20 & $\sim 10$ & 11 & 54 & 103 \\
\hline 3 & 50 & 20 & $\sim 50$ & 12 & 53 & n.o. ${ }^{f}$ \\
\hline 4 & 60 & 20 & $\sim 80$ & 13 & 53 & n.o. ${ }^{f}$ \\
\hline 5 & 80 & 60 & 100 & 20 & 54 & n.o. ${ }^{f}$ \\
\hline
\end{tabular}

${ }^{a}$ Reaction conditions: $10 \mathrm{~g}$ of 1,2 polybutadiene latex (6 wt. $\%$ polymer content), $20 \mathrm{~g}$ of water, ${ }^{b}$ Estimated by IR spectroscopy, $c f$. experimental section for details. ${ }^{c}$ Volume average particle size as determined by DLS. ${ }^{d}$ Determined on dialyzed dispersions, concentrated/diluted to a polymer solids content of $1.9 \mathrm{~g} \mathrm{~L} \quad{ }^{1}$. ${ }^{e}$ Determined by DSC. ${ }^{f}$ No $T_{\mathrm{m}}$ observed.

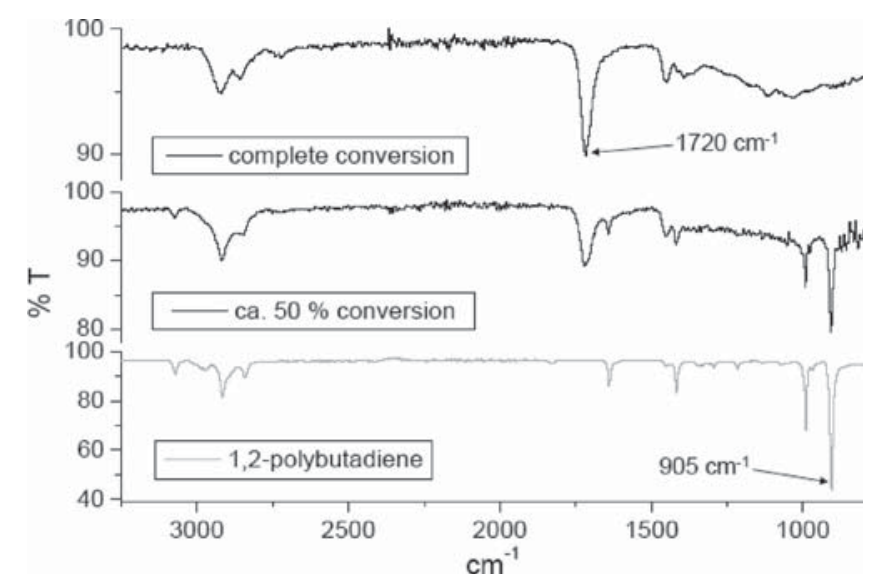

Figure 1. IR spectra of 1,2 polybutadiene starting material (bottom), and completely (top) and ca. 50\% converted (center) products.

during particle functionalization with $\mathrm{CO} / \mathrm{H}_{2}$, hydrogenation occurs at the most as a minor side reaction.

Differential scanning calorimetry (DSC) measurements were performed on the isolated polymers, obtained by precipitation from the dispersions. The semicrystalline syndiotactic 1,2-polybutadiene starting material, prepared in microemulsion, exhibits a $T_{\mathrm{m}}$ around $154{ }^{\circ} \mathrm{C}$. Hydroformylation results in a reduction of crystallinity, as expected. The $10 \%$ modified polymer exhibits a weak melting peak around $103{ }^{\circ} \mathrm{C}$. Higher conversions afford noncrystalline material as confirmed by powder X-ray diffraction (Figure 2). Nevertheless, all isolated polyaldehydes were insoluble in organic solvents. ${ }^{13}$ For polyaldehydes, aldol condensation can result in extensive cross-linking, and such reactions are enhanced by the high polymer concentration in particles.

Transmission electron microscopy (TEM; Figure 3) and scanning force microscopy (AFM; Supporting Information) on isolated particles confirm the small particle sizes determined by DLS. In both TEM and AFM, the 1,2-polybutadiene particles appear compact but not spherical, as expected for very small crystalline polymer particles (cf. Supporting Information). In contrast, fully functionalized particles appear smoother. However, they retain their particle character and do not flow (or aggregate) despite their amorphous character, which is in line with the particles being cross-linked.

(13) Solvents studied: trichloromethane, 1,1,2,2-tetrachloroethane- $d_{2}, 1,2,4$-trichlorobenzene, toluene, THF, DMSO, methanol, acetone.

(14) (a) Nakajima, A. Bull. Chem. Soc. Jpn. 1971, 44, 3272 3277. (b) Kalyanasundaram, K.; Thomas, J. K. J. Am. Chem. Soc. 1977, 99, 2039 2044. (c) Dong, D. C.; Winnik, M. A. Photochem. Photobiol. 1982, 35, 17 21. (d) Dong, D. C.; Winnik, M. A. Can. J. Chem. 1984, 62, 2560 2565. (e) Street, K. W.Jr.; Acree, W. E.Jr. Analyst 1986, 111, 1197 1201. (f) Tong, Q.; Krumova, M.; GottkerSchnetmann, I.; Mecking, S. Langmuir 2008, 24, 2341 2347. (g) For a detailed discussion of measures for solvent polarity, see: Reichardt, C. Solvents and Solvent Effects in Organic Chemistry, 3rd ed.; Wiley-VCH: Weinheim, 2003.

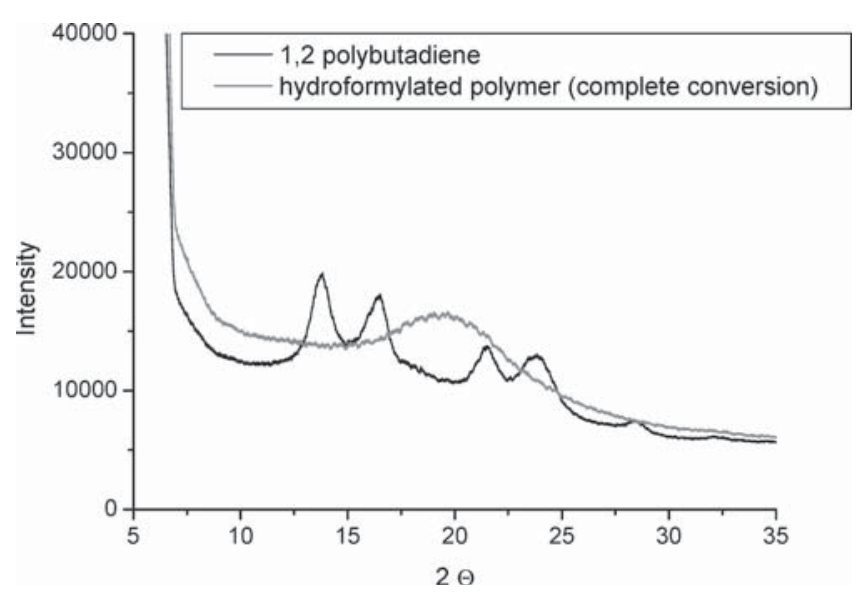

Figure 2. Powder $X$ ray diffraction of 1,2 polybutadiene (entry 1) and completely converted, hydroformylated polymer (entry 5).

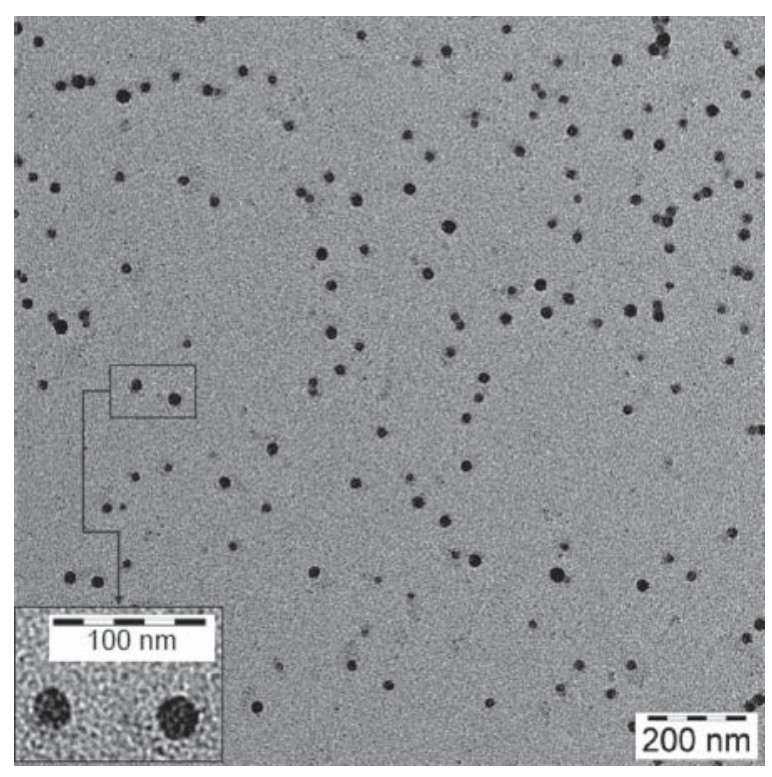

Figure 3. TEM image of fully hydroformylated nanoparticles.

The interaction of pyrene with nanoparticles of variable degree of hydroformylation was studied as a measure for their polarity. The relative intensity of two bands in the fluorescence spectra, $\mathrm{I}_{3} / \mathrm{I}_{1}$ is a proven measure for the polarity of the surrounding medium experienced by this fluorophore. ${ }^{14}$

Typical values of $I_{3} / I_{1}$ ratios in pyrene solutions are 0.63 for water, 1.07 for pentanol, and 1.65 for $n$-hexane. ${ }^{14 \mathrm{~b}}$ Emission spectra of pyrene were recorded over a range of particle concentrations and pyrene to polymer ratios (cf. Supporting Information). In all cases studied, the $\mathrm{I}_{3} / \mathrm{I}_{1}$ ratio increases gradually with 

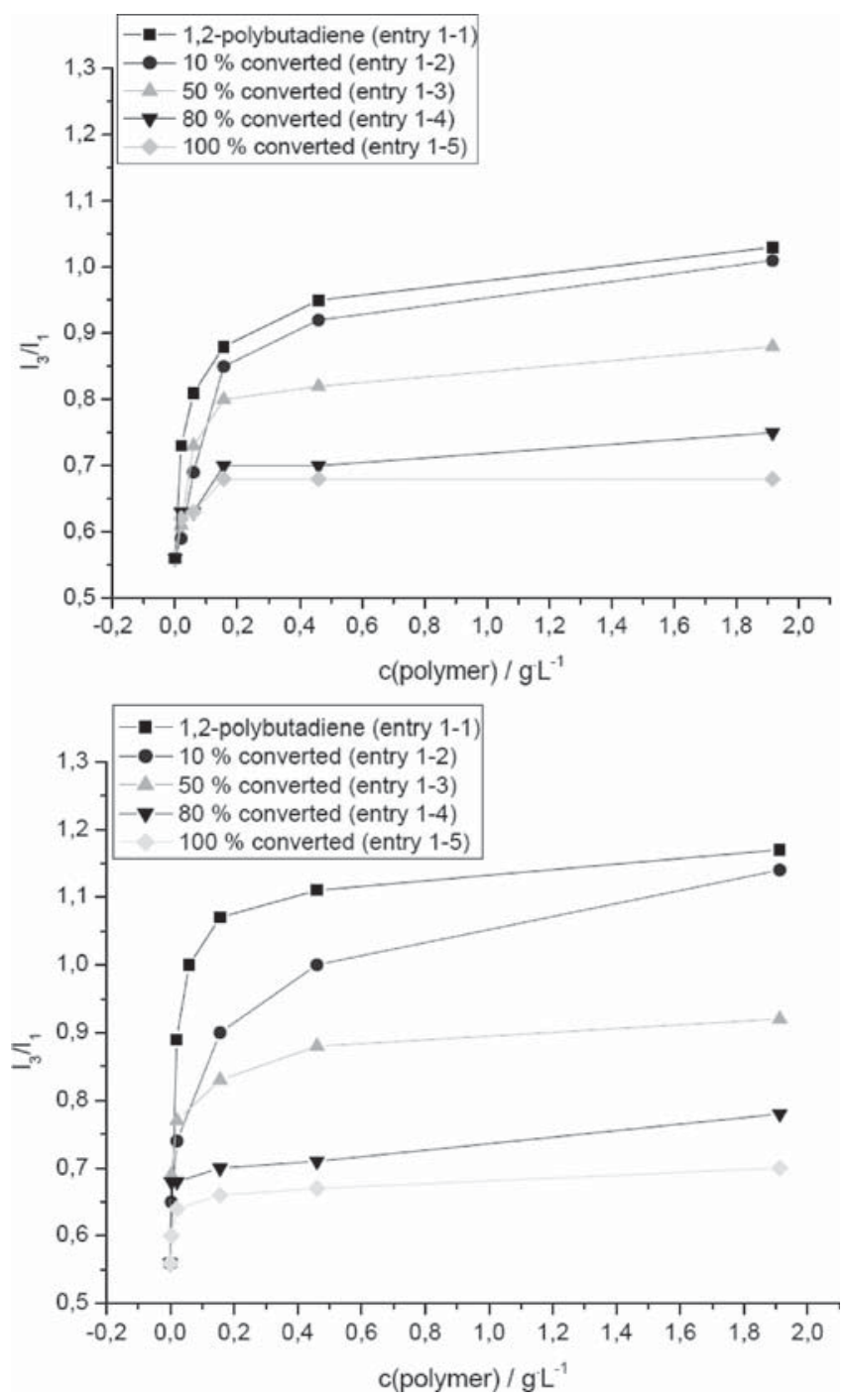

Figure 4. $I_{3} / I_{1}$ ratio of pyrene fluorescence versus polymer con centration of dispersions without quencher (top) and with quench er (bottom).

the concentration of polymer (Figure 4 and Supporting Information). As indicated, pyrene is slightly water-soluble and neat aqueous solutions also exhibit fluorescence. The overall signal therefore can also contain contributions from the aqueous phase. The portion of pyrene present in the nanoparticles increases with increasing polymer nanoparticle number density. A water-soluble quencher, diethylaminoethanol (DAE), selectively quenches the contribution from the aqueous phase. In the presence of this quencher, increased $I_{3} / I_{1}$ ratio are observed vs the absence of quencher. This confirms that the pyrene probe is indeed taken up by the nanoparticles, where it is not accessible; also the $\mathrm{I}_{3} / \mathrm{I}_{1}$ ratios determined in the presence of quencher are a more direct measure of the polarity of the nanoparticles. Note that the $\mathrm{I}_{3} / \mathrm{I}_{1}$ ratios found for the apolar 1,2-polybutadiene nanoparticles are significantly lower than those observed for hexane as a hydrocarbon solvent, similar to observations on semicrystalline polyethylene nanoparticles; this is likely due to location of the probe at the periphery of these crystalline particles, where it possibly senses the aqueous environment to a certain extent. ${ }^{14 \mathrm{f}}$

As an essence of these fluorescence studies, in all cases, the $\mathrm{I}_{3} / \mathrm{I}_{1}$ ratios observed with polymer nanoparticles of variable degrees of hydroformylation under otherwise identical conditions demonstrate that indeed the pyrene probe molecules experience an increasingly polar environment with increased content of carbonyl groups.

\section{Summary and Conclusion}

In summary, very small nanoparticles $(<20 \mathrm{~nm})$ with adjustable carbonyl content are accessible by hydroformylation of 1,2-polybutadiene in the form of aqueous dispersions. This represents a convenient route employing readily available starting materials (butadiene, $\mathrm{CO}$ and $\mathrm{H}_{2}$ ) to nanoparticles in this size range with a broadly adjustable and controllable polarity. The latter was demonstrated by fluorescence studies of the environment experienced by pyrene as a probe molecule.

Acknowledgment. Financial support by the BMBF (project 03X5505) is gratefully acknowledged. S.M. is indebted to the Fonds der Chemischen Industrie. M.C.M.-M. was supported by a postdoctoral fellowship by the Spanish Ministerio de Ciencia e Innovacion. We thank Lars Bolk for DSC and Carles Lizandara-Pueyo for powder X-ray diffraction experiments.

Supporting Information Available: TEM, AFM, DLS, and fluorescence data. This material is available free of charge via the Internet at http://pubs.acs.org. 